\title{
硬碳材料电极首周嵌钠过程的电化学阻抗谱研究
}

\author{
渠璐平任娅王宁史月丽庄全超* \\ (中国矿业大学材料科学与工程学院锂离子电池实验室 江苏省高效储能技术与装备工程实验室 徐州 221116)
}

\begin{abstract}
摘要 运用电化学阻抗谱(EIS)研究了硬碳材料电极嵌钠的过程, 发现 EIS 谱由两个半圆和一条斜线组成, 两个半圆可 归因于接触阻抗和钠离子通过固体电解质界面膜(SEI 膜)扩散的过程和电荷传递的过程, 斜线域则反映了钠离子在硬 碳材料颗粒内部的固态扩散相关的斜线. 通过选取适当的等效电路, 对实验结果进行拟合, 可以得出硬碳电极首周嵌 钠过程中 SEI 膜电阻、电子电阻等随电极极化电位的变化规律.
\end{abstract}

关键词 硬碳; 电解液体系; 钠离子电池; 电化学性能

\section{Electrochemical Impedance Spectroscopy Study on the First Sodium Insertion Process of Hard Carbon Material Electrode}

\author{
Qu, Luping Ren, Tong Wang, Ning Shi, Yueli Zhuang, Quanchao* \\ (Lithium Ion Battery Laboratory, Engineering Laboratory of High Efficiency Energy Storage Technology \& Equipments, \\ Jiangsu Provincial, School of Materials Science \& Engineering, China University of Mining \& Technology, Xuzhou 221116)
}

\begin{abstract}
In this study, electrochemical impedance spectroscopy (EIS) combined with cyclic volt-ampere (CV), charge-discharge measurement and scanning electron microscope were used. The electrode interface characteristics of hard carbon electrodes for sodium ion batteries in $1 \mathrm{~mol} / \mathrm{L} \mathrm{NaClO}_{4}$-EC:DEC and $1 \mathrm{~mol} / \mathrm{L}-\mathrm{NaClO}_{4}$-EC:DEC:PC electrolyte systems were discussed. The hard carbon material electrode is composed of $80 \mathrm{wt} \%$ active material, $10 \mathrm{wt} \%$ PVDF-HFP adhesive and $10 \mathrm{wt} \%$ conductive carbon black. The charge and discharge performance was tested with 2032 button battery and metal sodium sheet as counter electrode, the charge and discharge rate was $0.1 \mathrm{C}$, and the cut-off voltage was $0 \sim 3 \mathrm{~V}$. The three-electrode glass cell system was used for CV and EIS test, and the metal sodium sheet was used as the reference and auxiliary electrode. In the CV test, the scanning speed is $1 \mathrm{mV} / \mathrm{s}$, EIS and the frequency scanning range is $10^{5}$ to $10^{-2} \mathrm{~Hz}$. The amplitude of AC signal applied by $2 \mathrm{mV}$ is $5 \mathrm{mV}$. The electrochemical impedance spectra obtained in the experiment were simulated by Zview software. The results of $\mathrm{CV}$ show that the intercalation process of sodium ion in hard carbon materials is mainly divided into two steps, that is, the filling process of sodium ion in nano-pores, the intercalation of sodium ion in graphene layer and the adsorption and desorption of sodium ion on the surface or defect. The filling process of sodium ion in the nanoporous is accompanied by the formation of solid electrolyte interface (SEI) film on the surface of the electrode. The results of electrochemical impedance spectroscopy show that the spectrum consists of two semicircles and a oblique line, which can be attributed to the contact impedance, the diffusion of sodium ions through SEI film and the process of charge transfer. The oblique domain reflects the oblique line related to the solid diffusion of sodium ion in the particles of hard carbon materials. By selecting the appropriate equivalent circuit and fitting the experimental results, we can get the variation of SEI film resistance and electron resistance with the electrode polarization potential in the process of sodium insertion in the first week of the hard carbon electrode.
\end{abstract}

Keywords hard carbon; electrolyte system; sodium ion battery; electrochemical property

\section{1 引言}

钠离子电池具有资源丰富、成本低廉、环境友好等 优势, 有望取代锂离子电池在大规模储能系统中获得应 用 ${ }^{[1 \sim 3]}$, 然而合适的实用化负极材料的缺乏直接限制了 其商业化进程. 由于价格低廉、易于制备和化学性质稳 定等特点, 自 1990 年锂离子电池开始商用以来, 石墨一 直是最主要的商品化负极材料. 然而由于其层间间距较 小, 导致钠离子在石墨层间脱嵌困难, 储钠能力较差 ${ }^{[4]}$. 目前, 已研究过的钠离子电池负极体系包括碳材料、合
金类、氧化物、有机化合物等, 其中, 硬碳由于具有较 低的嵌钠平台 $(\approx 0.1 \mathrm{~V})$ 和较高的比容量 $(300 \sim 400$ $\mathrm{mAh} / \mathrm{g}$ ), 且来源丰富, 被认为是最有希望推动钠离子电 池产业化的负极材料 ${ }^{[5 \sim 8]}$.

在锂离子电池中的研究结果表明, 无论是石墨负极 材料还是正极材料, 在首周充放电过程中甚至是仅仅由 于电极活性材料与电解液的接触, 就会在电极/电解质 界面形成固体电解质界面膜(SEI 膜), SEI 膜的形成对其 电化学性能起着极其关键的作用 ${ }^{[0 \sim 12]}$. 已有研究结果表

* E-mail: zhuangquanchao@126.com

Received March 29, 2019; published June 12, 2019. 
明, 在钠离子电池中电极表面同样存在 SEI 膜, 并起到 类似于锂离子电池中 SEI 膜的作用 ${ }^{[7]}$. 电化学阻抗谱 (EIS)是研究电极界面性质最有力的工具之一, EIS 能够 根据电化学反应过程中每一步骤弛豫时间常数的不同, 在较宽频率范围对其表征, 进而得到了 SEI 膜的形成机 制以及电池电化学性能的衰减机理 ${ }^{[13 \sim 19]}$. 钠离子电池 与锂离子电池具有相似的工作机理, EIS 必然会在钠离 子电池电极界面性质研究中起到重要作用, 但这方面的 研究工作仍然鲜见文献报道.

因此, 在本研究工作中, 运用电化学阻抗谱并结合 循环伏安 $(\mathrm{CV})$ 、充放电测试以及扫描电子显微镜等研究 方法, 探讨了钠离子电池硬碳材料电极在 $1 \mathrm{~mol} / \mathrm{L}$ $\mathrm{NaClO}_{4}-\mathrm{EC}: \mathrm{DEC}\left(\right.$ 体积比为 $1: 1$ ) 和 $1 \mathrm{~mol} / \mathrm{L}^{-} \mathrm{NaClO}_{4}-$ EC:DEC:PC(体积比 $1: 1: 1)$ 两种电解液体系中的电极 界面特性.

\section{2 结果与讨论}

\subsection{XRD 测试结果}

图 1 是硬碳材料 XRD 图谱. 从中可以看出, 样品的 XRD 图谱在大约 $23^{\circ}$ 和 $44^{\circ}$ 左右都存在明显的漫包峰, 对应于无定型碳的特征峰 ${ }^{[20]}$, 表明本实验中使用的硬 碳材料为非晶态结构. 图中 $23^{\circ}$ 左右的衍射峰对应的是 (002)晶面, 44左右的衍射峰对应的(100)晶面. 计算可 得, (002)的晶面间距为 $0.3863 \mathrm{~nm}$, 这对钠离子的嵌入 与脱出是有利的 ${ }^{[21]}$.

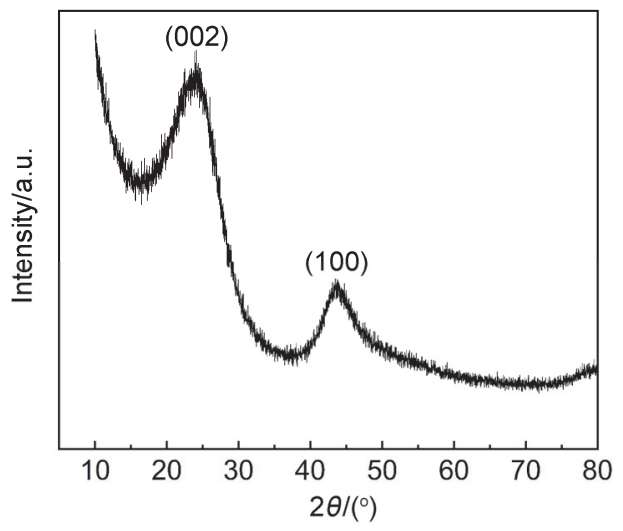

图 1 硬碳原料的 XRD 图

Figure 1 XRD diagram of hard carbon materials

\subsection{CV 和充放电研究结果}

图 $2 \mathrm{a}$ 和 $2 \mathrm{~b}$ 为硬碳材料电极在 $1 \mathrm{~mol} / \mathrm{L} \mathrm{NaClO}_{4}$ EC:DEC 和 $1 \mathrm{~mol} / \mathrm{L} \mathrm{NaClO}_{4}$-EC:DEC:PC 中的 CV 图. 可 以看出, 在第一次负向扫描过程中, 无论是在 $1 \mathrm{~mol} / \mathrm{L}$ $\mathrm{NaClO}_{4}$-EC:DEC 还是 $1 \mathrm{~mol} / \mathrm{L} \mathrm{NaClO}_{4}$-EC:DEC:PC 中, 硬碳材料电极的 $\mathrm{CV}$ 曲线中均存在两个还原电流峰, 即 $0.7 \mathrm{~V}$ 附近的 $\alpha\left(\alpha^{\prime}\right)$ 峰和 $0.0 \mathrm{~V}$ 附近的 $\beta\left(\beta^{\prime}\right)$ 峰. 在第一周 扫描过后, 随后的扫描过程中峰 $\alpha$ 和 $\alpha^{\prime}$ 明显减小, 并呈
现出较好的可逆性, 显示峰 $\alpha$ 和 $\alpha^{\prime}$ 可能不仅与硬碳材料 电极表面生成 SEI 膜的过程相关还与钠离子在硬碳材料 中的嵌入过程有关. 在后续的扫描中, $\alpha\left(\alpha^{\prime}\right)$ 和 $\beta\left(\beta^{\prime}\right)$ 峰一 直存在. 在正向扫描过程中, 与负向扫描的 $\alpha\left(\alpha^{\prime}\right)$ 和 $\beta$ $\left(\beta^{\prime}\right)$ 还原电流峰相对应, 出现了 $\lambda\left(\lambda^{\prime}\right) 、 \theta\left(\theta^{\prime}\right)$ 两个氧化电 流峰, 呈现为两对氧化还原电流峰, 表明钠离子在硬碳 材料中的嵌入过程主要分为两步进行, 即 $\alpha\left(\alpha^{\prime}\right)$ 峰对应 于钠离子在纳米孔内的填充过程, $\beta\left(\beta^{\prime}\right)$ 峰归因于钠离子 在石墨烯层间的嵌入与脱出的过程 ${ }^{[22,23]}$. 此外, 硬碳材 料电极在 $1 \mathrm{~mol} / \mathrm{L} \mathrm{NaClO}_{4}-\mathrm{EC}: \mathrm{DEC}: \mathrm{PC}$ 中的首周不可逆 容量明显比硬碳在 $1 \mathrm{~mol} / \mathrm{L} \mathrm{NaClO}_{4}$-EC:DEC 低很多, 并 且循环伏安曲线表现出了较好的重合性, 表明在 1 $\mathrm{mol} / \mathrm{L} \mathrm{NaClO}_{4}-\mathrm{EC}+\mathrm{DEC}+\mathrm{PC}$ 的电解液体系中有更好的 电化学性能.
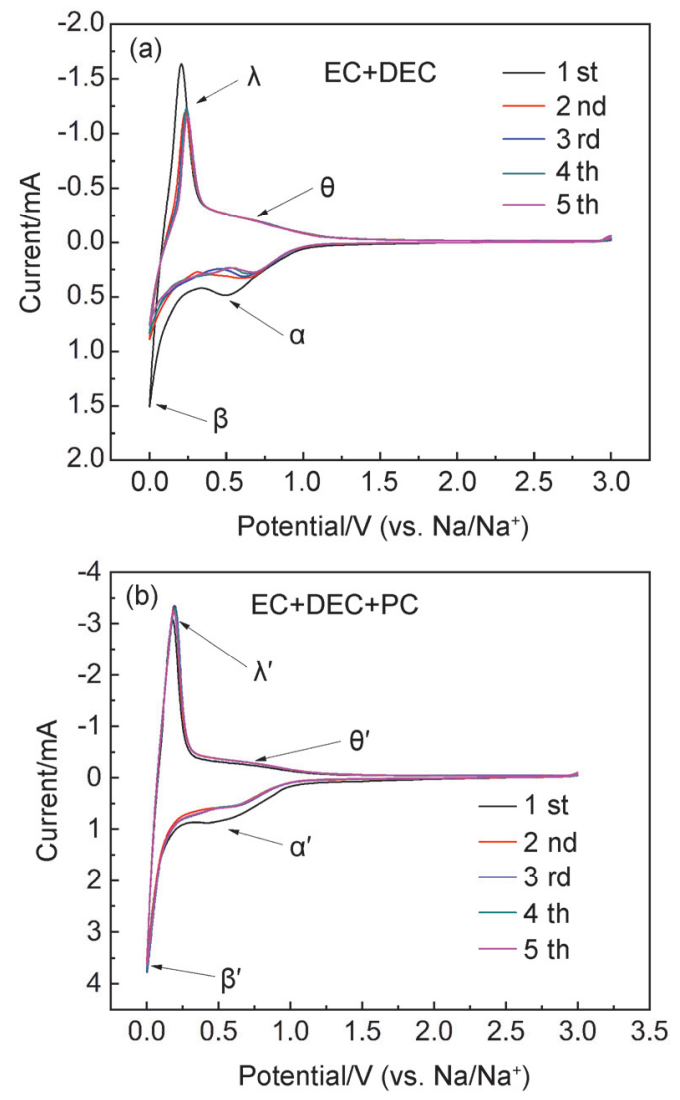

图 2 硬碳在 $1 \mathrm{~mol} / \mathrm{L} \mathrm{NaClO}_{4}$-EC+DEC (a)和 $1 \mathrm{~mol} / \mathrm{L} \mathrm{NaClO}$ - $\mathrm{EC}+$ $\mathrm{DEC}+\mathrm{PC}(\mathrm{b})$ 中的 $\mathrm{CV}$ 图

Figure2 $\mathrm{CV}$ diagrams of hard carbon in $1 \mathrm{~mol} / \mathrm{L} \mathrm{NaClO}_{4}-\mathrm{EC}+\mathrm{DEC}$ (a) and $1 \mathrm{~mol} / \mathrm{L} \mathrm{NaClO}_{4}-\mathrm{EC}+\mathrm{DEC}+\mathrm{PC}(\mathrm{b})$

图 $3 \mathrm{a}, 3 \mathrm{~b}$ 为硬碳材料电极在 $1 \mathrm{~mol} / \mathrm{L} \mathrm{NaClO}_{4}$ $\mathrm{EC}: \mathrm{DEC}$ 和 $1 \mathrm{~mol} / \mathrm{L} \mathrm{NaClO}_{4}-\mathrm{EC}: \mathrm{DEC}: \mathrm{PC}$ 中的首周充放 电曲线和循环性能曲线. 由首周充放电曲线可以看出, 硬碳材料电极充放电曲线主要包括 $0.7 \sim 0.2 \mathrm{~V}$ 之间的电 位斜坡区与 $0.2 \mathrm{~V}$ 以下的平台区两部分，这与 $\mathrm{CV}$ 研究 的结果相一致, 因此斜坡区峰对应于钠离子在纳米孔内 
的填充过程，而平台区则可归因于钠离子在石墨烯层间 的嵌入与脱出的过程 ${ }^{[22,23]}$. 硬碳材料电极在 $1 \mathrm{~mol} / \mathrm{L}$ $\mathrm{NaClO}_{4}-\mathrm{EC}: \mathrm{DEC}$ 中的首周放电容量为 $301.9 \mathrm{mAh} / \mathrm{g}$, 首 周的充电容量为 $230.7 \mathrm{mAh} / \mathrm{g}$, 由此可得硬碳电极的首 周库伦效率为 $76.4 \%$; 在 $1 \mathrm{~mol} / \mathrm{L} \mathrm{NaClO}_{4}$-EC:DEC:PC $(1: 1: 1, V: V: V)$ 中首周的放电容量为: $343.1 \mathrm{mAh} / \mathrm{g}$, 充电容量为 $240.0 \mathrm{mAh} / \mathrm{g}$, 由此可得硬碳电极的首周库 伦效率为 $70.0 \%$.
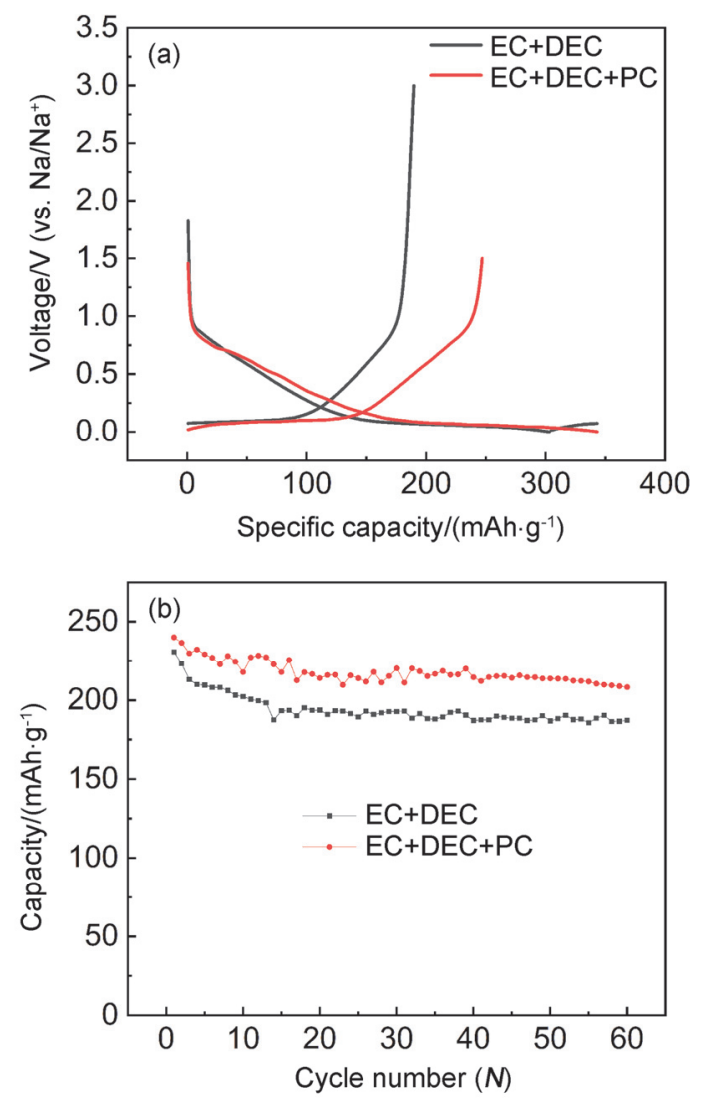

图 3 硬碳在 $1 \mathrm{~mol} / \mathrm{L} \mathrm{NaClO}_{4}-\mathrm{EC}+\mathrm{DEC}$ 和 $1 \mathrm{~mol} / \mathrm{L} \mathrm{NaClO}_{4}-\mathrm{EC}+\mathrm{DEC}$ $+\mathrm{PC}$ 中的首周充放电曲线( $(a)$ 和循环性能曲线 $(b)$

Figure 3 Charge-discharge characteristics (a) and cyclic performance (b) of hard carbon in $1 \mathrm{~mol} / \mathrm{L} \mathrm{NaClO}_{4}-\mathrm{EC}+\mathrm{DEC}$ and $1 \mathrm{~mol} / \mathrm{L}$ $\mathrm{NaClO}_{4}-\mathrm{EC}+\mathrm{DEC}+\mathrm{PC}$

虽然硬碳电极在 $1 \mathrm{~mol} / \mathrm{L} \mathrm{NaClO}_{4}-\mathrm{EC}+\mathrm{DEC}+\mathrm{PC}$ 库 伦效率低于在 $1 \mathrm{~mol} / \mathrm{L} \mathrm{NaClO}_{4}-\mathrm{EC}: \operatorname{DEC}(1: 1, V: V)$, 但是循环 60 周以后, 硬碳负极在 $1 \mathrm{~mol} / \mathrm{L} \mathrm{NaClO}_{4}$-EC: DEC $(1: 1, V / V)$ 电解液中的充电容量为 $188.1 \mathrm{mAh} / \mathrm{g}$, 充电保持率为 $81.5 \%$, 在 $1 \mathrm{~mol} / \mathrm{L} \mathrm{NaClO}_{4}$-EC:DEC:PC $(1: 1: 1, V: V: V)$ 的充电容量为 $208.6 \mathrm{mAh} / \mathrm{g}$, 充电保 持率为 $86.9 \%$. 因此可知, 一定量 PC 的加入可以提高硬 碳电极在钠离子电池循环过程的稳定性.

\subsection{SEM 研究结果}

图 4 为硬碳材料样品的 SEM 图. 可以看到, 本工作 所使用的硬碳材料是块状结构, 直径在 $5 \mathrm{~nm}$ 左右, 在经
历电化学扫描循环前, 可以清晰地观察到块状的硬碳材 料颗粒, 其表面比较光滑, 不存在原始 SEI 膜. 硬碳材 料电极在 $1 \mathrm{~mol} / \mathrm{L} \mathrm{NaClO}_{4}$-EC:DEC 电解液中经历电化学 扫描循环后，已完全看不到块状的硬碳材料颗粒，显示 在硬碳材料电极表面形成了一层较厚的 SEI 膜. 然而, 硬碳材料电极在 $1 \mathrm{~mol} / \mathrm{L} \mathrm{NaClO}_{4}-\mathrm{EC}: \mathrm{DEC}: \mathrm{PC}$ 电解液中 经历电化学扫描循环后, 虽然硬碳材料电极表面已变得 有些模糊，但仍能看到块状的硬碳材料颗粒，显示相比 于在 $1 \mathrm{~mol} / \mathrm{L} \mathrm{NaClO}_{4}-\mathrm{EC}: \mathrm{DEC}$ 电解液中, 硬碳材料电极 在 $1 \mathrm{~mol} / \mathrm{L} \mathrm{NaClO}_{4}-\mathrm{EC}: \mathrm{DEC}: \mathrm{PC}$ 解液中表面形成的 SEI 膜厚度要小一些.
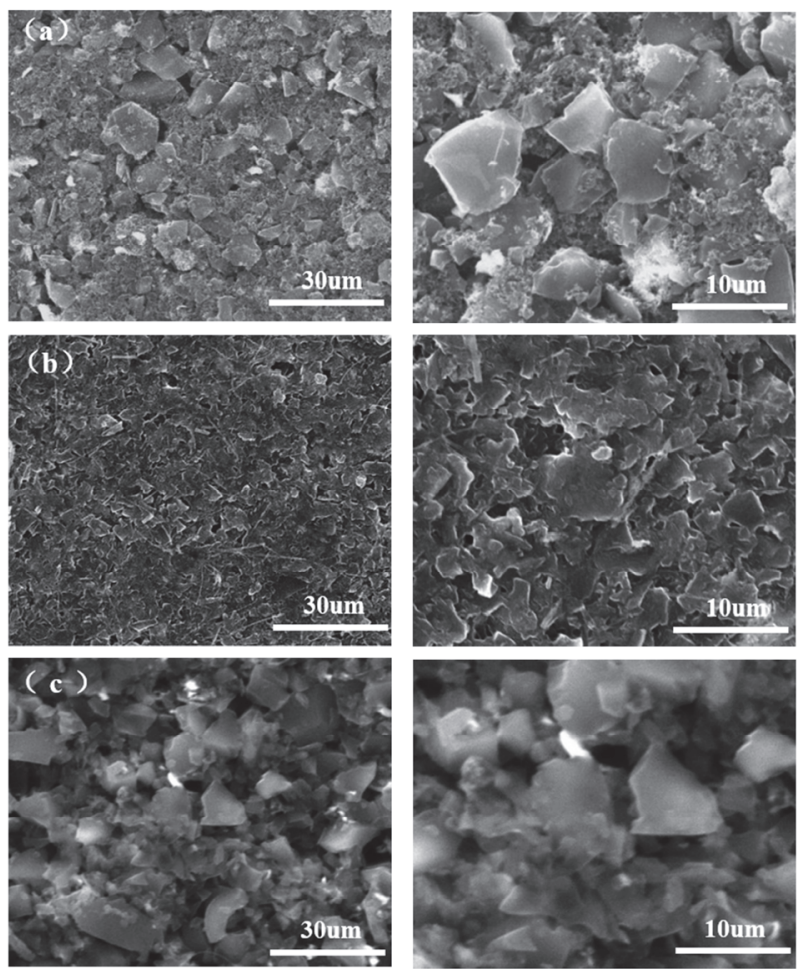

图 4 硬碳材料电极在经历电化学扫描循环前和在不同电解液中经历 电化学扫描循环后的 SEM 图

Figure 4 SEM diagram of hard carbon material electrode before electrochemical scanning cycle and after electrochemical scanning cycle in different electrolytes

(a) before electrochemical testing, (b) blank, (c) add PC after electrochemical testing

\subsection{EIS 研究结果}

图 5 为硬碳材料电极在 $1 \mathrm{~mol} / \mathrm{L} \mathrm{NaClO}_{4}-\mathrm{EC}: \mathrm{DEC}$ 和 $1 \mathrm{~mol} / \mathrm{L} \mathrm{NaClO}_{4}-\mathrm{EC}: \mathrm{DEC}: \mathrm{PC}$ 电解液中首周钠离子嵌入 过程中电极极化电位在 $3.0 \sim 0.1 \mathrm{~V}$ 时 EIS 的 Nyquist 图. 可以看到, 硬碳材料电极在 $1 \mathrm{~mol} / \mathrm{L} \mathrm{NaClO}_{4}-\mathrm{EC}: \mathrm{DEC}$ 和 $1 \mathrm{~mol} / \mathrm{L} \mathrm{NaClO}_{4}$-EC:DEC:PC 电解液中显示出相似的阻 抗谱特征和随电极极化电位变化的规律. 硬碳材料电极 在首周阴极极化过程中, 在开路电位 $(2.8 \mathrm{~V})$ 时 EIS 的 Nyquist 图由一高频区域小半圆(High-frequency semicir- 

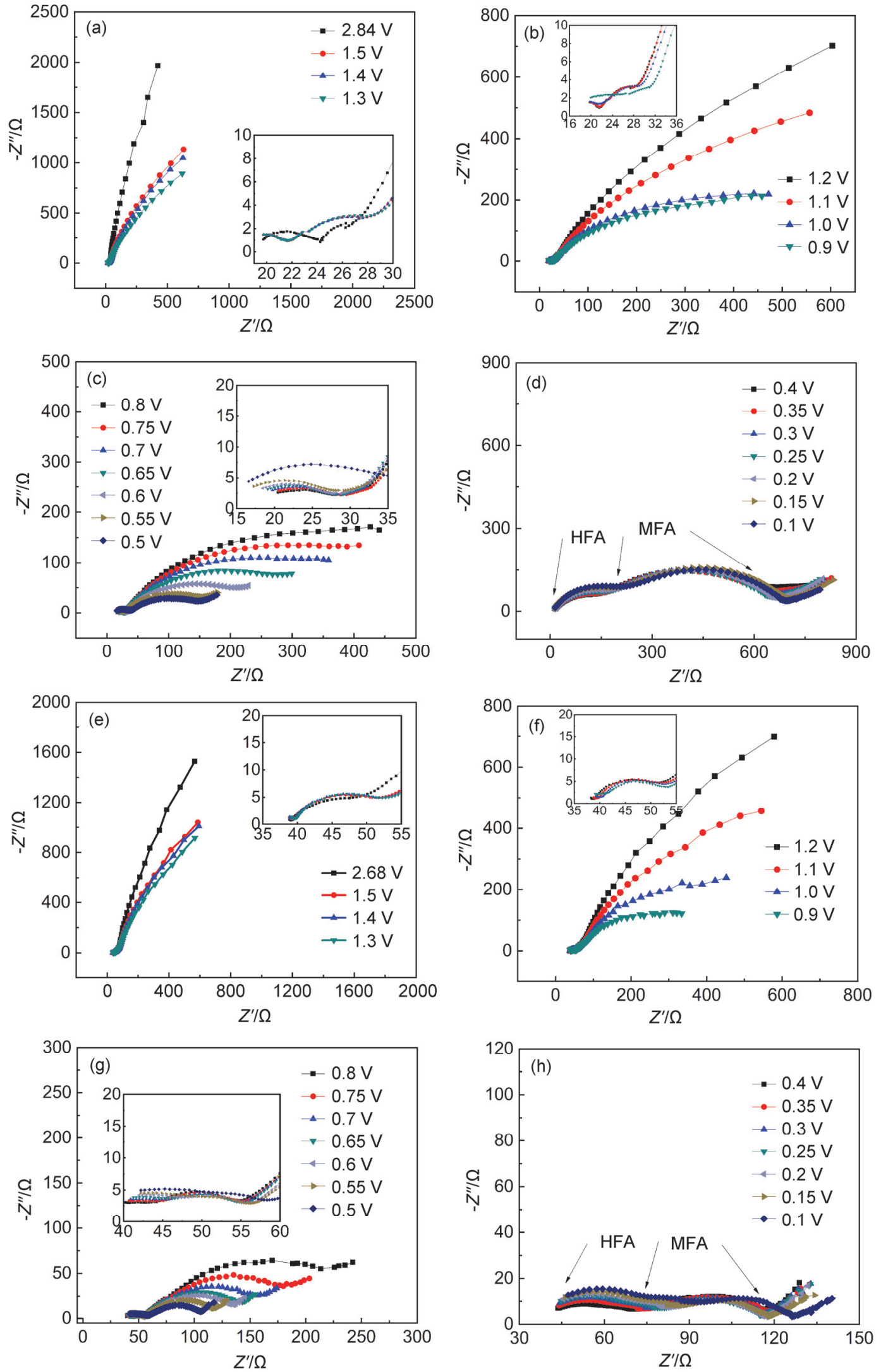

图 5 硬碳材料电极首周钠离子嵌入过程中电极极化电位在 3.0 0.1 V 时 EIS 的 Nyquist 图

Figure 5 Nyquist diagram of EIS at $3.0 \mathrm{~V}$ and $0.1 \mathrm{~V}$ during sodium ion intercalation in the first week of hard carbon material electrode $(\mathrm{a} \sim \mathrm{d}) 1 \mathrm{~mol} / \mathrm{L} \mathrm{NaClO}{ }_{4}-\mathrm{EC}+\mathrm{DEC},(\mathrm{e} \sim \mathrm{h}) 1 \mathrm{~mol} / \mathrm{L} \mathrm{NaClO}_{4}-\mathrm{EC}+\mathrm{DEC}+\mathrm{PC}$ 
cle, HFS)和低频区域的一段圆弧组成. 通过 CV 和 SEM 研究结果可知, 硬碳材料表面不存在原始 SEI 膜, 而且 在开路电位时也不存在与 SEI 膜成膜相关的过程, 因此 此时高频区域内的小半圆只能归因于电解液和硬碳材 料、硬碳材料颗粒和硬碳材料颗粒以及硬碳材料颗粒和 集流体之间的接触问题 ${ }^{[24]}$, 低频区域的圆弧反映的是 电极阻滞特性 ${ }^{[25]}$. 硬碳材料电极在两种电解液中 $1.3 \mathrm{~V}$ 以上阻抗的特征与开路电位一致, 当电位进一步降低 时, 可以看到低频区域内的圆弧不断下弯, 并且在中频 区域内开始形成一个新的半圆. 当电极极化电位进一步 降低至 $0.8 \mathrm{~V}$ 时(在 $1 \mathrm{~mol} / \mathrm{L} \mathrm{NaClO}_{4}-\mathrm{EC}: \mathrm{DEC}: \mathrm{PC}$ 电解液 中, 在 $1 \mathrm{~mol} / \mathrm{L} \mathrm{NaClO}_{4}-\mathrm{EC}: \mathrm{DEC}$ 为 $0.65 \mathrm{~V}$ ), 硬碳材料电 极 EIS 的 Nyquist 图由高频区域内的半圆(HFS)、中频区 域内的半圆(Medium-frequency semicircle, MFS)以及低 频区的斜线组成. 在随后的电极极化电位进一步降低过 程中, 硬碳材料电极 EIS 谱特征未再发生明显的改变. 上述硬碳材料电极在首周钠离子嵌入过程中的 EIS 谱特 征及其随电极极化电位的变化规律与石墨电极在首周 锂离子嵌入过程中 EIS 谱特征及其随电极极化电位的变 化规律基本相同 ${ }^{[13,26]}$, 因此, HFS 可归因于接触阻抗和 钠离子通过 SEI 膜扩散过程相关的半圆, MFS 是与电荷 传递过程相关的半圆，低频区的斜线域则反映了钠离子 在硬碳材料颗粒内部的固态扩散相关的斜线.

根据本实验结果, 提出如图 6 所示硬碳材料电极首 周钠离子嵌入过程中 EIS 等效电路. 为了进一步研究在 电解液体系对钠离子硬碳负极电化学性能的影响, 使用 图 6 所示的拟合电路对实验获得的阻抗数据进行拟合. 图中的 $R_{\mathrm{s}}$ 代表了系统的欧姆电阻, $R_{\mathrm{SEI}}$ 和 $R_{\mathrm{ct}}$ 分别代表电 极中与 SEI 膜相关电阻(包含接触电阻)和与电荷传递相 关电阻, 用恒相角元件(CPE) $Q_{\mathrm{SEI}}$ 代表 SEI 膜电容, 用 $Q_{\mathrm{dl}}$ 代表溶液的双电层电容, 浓差阻抗则用 $Q_{\mathrm{D}}$ 来表示. 拟合过程中根据 EIS 谱特征调整 $Q$ 和 $R$ 等原件实现对 不同电位下 EIS 谱的拟合.

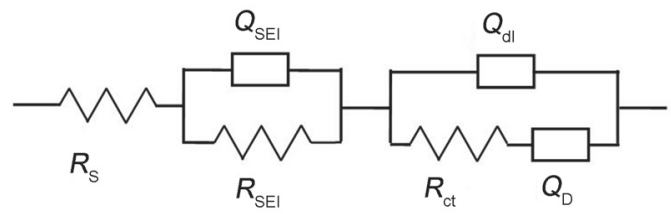

图 6 拟合 EIS 所需的等效电路

Figure 6 Equivalent circuit proposed for fitting impedance spectra

图 7 为硬碳材料电极在 $1 \mathrm{~mol} / \mathrm{L} \mathrm{NaClO}_{4}-\mathrm{EC}: \mathrm{DEC}$ 和 $1 \mathrm{~mol} / \mathrm{L} \mathrm{NaClO}_{4}$-EC:DEC:PC 电解液中 $R_{\mathrm{SEI}}$ 随电极极化 电位的变化曲线. 可以看出, 在两种不同的电解液体系 中, $R_{\mathrm{SEI}}$ 随电极极化电位的变化呈现相近的变化趋势. 当电极极化电位在 $2.8 \mathrm{~V}$ 至 $1.0 \mathrm{~V}$ 之间, 随电极极化电位 的降低, $R_{\mathrm{SEI}}$ 大小基本保持不变, 表明此时硬碳材料电
极表面不存在明显的 SEI 膜形成过程, $R_{\mathrm{SEI}}$ 主要归因于 接触阻抗问题. 当电极极化电位在 $1.0 \sim 0.6 \mathrm{~V}$ 之间时, 随电极极化电位的降低, $R_{\mathrm{SEI}}$ 缓慢增大, 显示在此电位 区间, SEI 膜已经在硬碳材料颗粒表面开始形成. 当电 极极化电位从 $0.6 \mathrm{~V}$ 降低到 $0.1 \mathrm{~V}$ 过程中, $R_{\mathrm{SEI}}$ 快速增大, 表明硬碳材料颗粒表面 SEI 膜厚度快速增加. 相比于在 $1 \mathrm{~mol} / \mathrm{L} \mathrm{NaClO}_{4}$-EC:DEC 电解液中, 在 $1 \mathrm{~mol} / \mathrm{L} \mathrm{NaClO}_{4}$ $\mathrm{EC}: \mathrm{DEC}: \mathrm{PC}$ 电解液中 $0.5 \sim 0.1 \mathrm{~V}$ 之间 $R_{\mathrm{SEI}}$ 增长速度明显 受到抑制, 显示在 $1 \mathrm{~mol} / \mathrm{L} \mathrm{NaClO}_{4}-\mathrm{EC}: \mathrm{DEC}$ 中加入 PC 能够明显抑制硬碳材料颗粒表面 SEI 膜厚度快速增加的 趋势, 进而改善硬碳材料电极的电化学循环性能, 这与 充放电研究结果是一致的. 然而与石墨电极在首周嵌锂 过程中 $R_{\mathrm{SEI}}$ 随电极极化电位变化趋势相比 ${ }^{[27,28]}$, 硬碳材 料电极无论是在 $1 \mathrm{~mol} / \mathrm{L} \mathrm{NaClO}_{4}-\mathrm{EC}: \mathrm{DEC}$ 还是 $1 \mathrm{~mol} / \mathrm{L}$ $\mathrm{NaClO}_{4}-\mathrm{EC}: \mathrm{DEC}: \mathrm{PC}$ 电解液中, 其表面在首周阴极极化 过程中形成的 SEI 膜均无法对其实现有效的钝化.

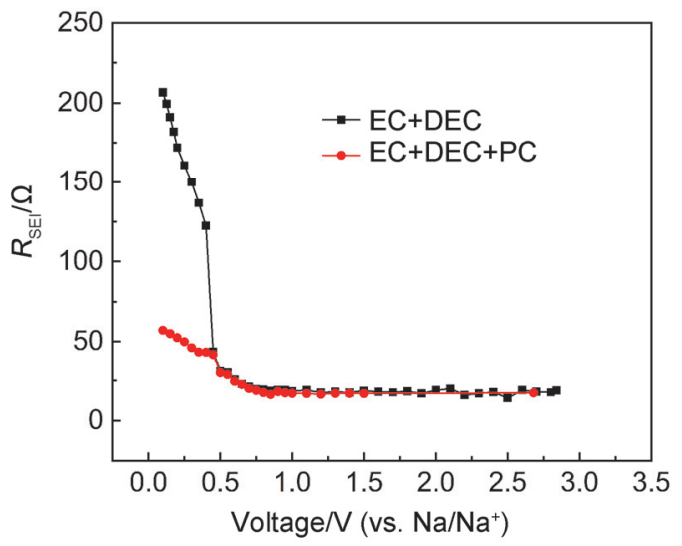

图 7 在添加和未添加 $\mathrm{PC}$ 电解液中 $R_{\mathrm{SEI}}$ 随电极极化电位的变化

Figure 7 Variations of $R_{\text {SEI }}$ with the decrease of electrode polarization potential

图 8 为硬碳材料电极在 $1 \mathrm{~mol} / \mathrm{L} \mathrm{NaClO}_{4}-\mathrm{EC}: \mathrm{DEC}$ 和 $1 \mathrm{~mol} / \mathrm{L} \mathrm{NaClO}_{4}-\mathrm{EC}: \mathrm{DEC}: \mathrm{PC}$ 电解液中 $R_{\mathrm{ct}}$ 随电极极化电 位的变化曲线. 可以看出, 在 $1 \mathrm{~mol} / \mathrm{L} \mathrm{NaClO}_{4}$-EC:DEC 电解液中, 当电极极化电位在 $0.9 \mathrm{~V}$ 至 $0.4 \mathrm{~V}$ 之间, 随电 极极化电位的降低, $R_{\mathrm{ct}}$ 先减小而后增大; 当电极极化电 位从 $0.4 \mathrm{~V}$ 降低到 $0.1 \mathrm{~V}$ 过程中, $R_{\mathrm{ct}}$ 逐渐减小. 上述结果 表明, 钠离子在硬碳材料中的嵌入过程主要分为两步进 行. 然而, 在 $1 \mathrm{~mol} / \mathrm{L} \mathrm{NaClO}_{4}-\mathrm{EC}: \mathrm{DEC}: \mathrm{PC}$ 电解液中, 当 电极极化电位在 $0.9 \mathrm{~V}$ 至 $0.5 \mathrm{~V}$ 之间, 随电极极化电位 的降低, $R_{\mathrm{ct}}$ 迅速减小; 当电极极化电位从 $0.5 \mathrm{~V}$ 降低到 $0.1 \mathrm{~V}$ 过程中, $R_{\mathrm{ct}}$ 基本保持不变, 呈现出与石墨电极在首 周嵌锂过程中 $R_{\mathrm{ct}}$ 随电极极化电位变化相近的趋势 ${ }^{[27]}$. 此外, 在 $1 \mathrm{~mol} / \mathrm{L} \mathrm{NaClO}_{4}-\mathrm{EC}: \mathrm{DEC}: \mathrm{PC}$ 电解液中, $R_{\mathrm{ct}}$ 的值 整体明显小于在 $1 \mathrm{~mol} / \mathrm{L} \mathrm{NaClO}_{4}-\mathrm{EC}: \mathrm{DEC}$ 电解液中, 特 别是在大量钠离子开始嵌入过程中 $(0.5 \mathrm{~V}$ 以下 $)$. 上述结 
果表明, 在 $1 \mathrm{~mol} / \mathrm{L} \mathrm{NaClO}_{4}-\mathrm{EC}: \mathrm{DEC}$ 中加入 $\mathrm{PC}$ 不仅能 够有效降低钠离子嵌入硬碳材料过程中 $R_{\mathrm{ct}}$ 的值, 而且 能够使两步嵌入过程转变为一步嵌入过程, 使钠离子在 硬碳材料中的嵌入过程变得更容易.

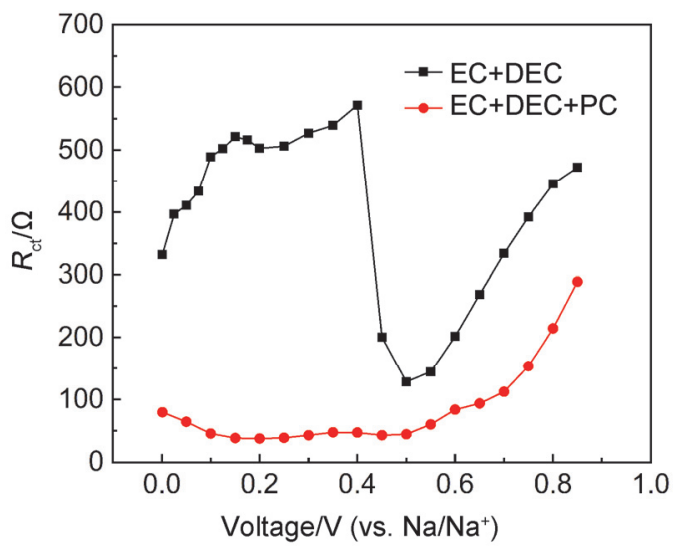

图 8 在添加和未添加 $\mathrm{PC}$ 电解液中 $R_{\mathrm{ct}}$ 随电极极化电位的变化 Figure 8 Variations of $R_{\mathrm{ct}}$ with the decrease of electrode polarization potential

\section{3 结论}

运用电化学阻抗谱并结合循环伏安 $(\mathrm{CV})$ 、充放电测 试以及扫描电子显微镜等研究方法, 研究了钠离子电池 硬碳材料电极在 $1 \mathrm{~mol} / \mathrm{L} \mathrm{NaClO}_{4}$-EC:DEC 和 $1 \mathrm{~mol} / \mathrm{L}$ $\mathrm{NaClO}_{4}-\mathrm{EC}: \mathrm{DEC}: \mathrm{PC}$ 两种电解液体系中的电极界面特 性.

$\mathrm{CV}$ 研究结果表明, 钠离子在硬碳材料中的嵌入过 程主要分为两步进行, 即钠离子在纳米孔内的填充过程 和钠离子在石墨烯层间的嵌插以及在表面或缺陷处的 吸脱附, 在钠离子在纳米孔内的填充过程中, 同时伴有 电极表面开始形成 SEI 膜的过程.

充放电结果表明, 循环 60 周以后, 硬碳负极在 1 $\mathrm{mol} / \mathrm{L} \mathrm{NaClO}_{4}$-EC:DEC $(1: 1, V: V)$ 电解液中的充电容 量为 $188.1 \mathrm{mAh} / \mathrm{g}$, 充电保持率为 $81.5 \%$, 硬碳电极在 1 $\mathrm{mol} / \mathrm{L} \mathrm{NaClO}_{4}$-EC: DEC:PC $(1: 1: 1, V: V: V)$ 的充电 容量为 $208.6 \mathrm{mAh} / \mathrm{g}$, 充电保持率为 $86.9 \%$.

SEM 研究结果表明, 相比于在 $1 \mathrm{~mol} / \mathrm{L} \mathrm{NaClO}_{4}$ EC:DEC 电解液中, 硬碳材料电极在 $1 \mathrm{~mol} / \mathrm{L} \mathrm{NaClO}_{4}-$ EC:DEC:PC 解液中表面形成的 SEI 膜厚度要小一些. EIS 研究结果表明, 硬碳电极在 $1 \mathrm{~mol} / \mathrm{L} \mathrm{NaClO}_{4}$-EC+ $\mathrm{DEC}+\mathrm{PC}$ 中经过阻抗测试并拟合后发现, 在 $1 \mathrm{~mol} / \mathrm{L}$ $\mathrm{NaClO}_{4}-\mathrm{EC}+\mathrm{DEC}+\mathrm{PC}$ 中得出的 $R_{\mathrm{SEI}}$ 与 $R_{\mathrm{ct}}$ 阻值更小.

\section{4 实验部分}

硬碳材料电极按 $80 \%$ 的活性材料(日本 ATEC 公 司)、10\%的 PVDF-HFP 粘合剂(Kynar FLEX LBG, Elf-atochem, USA)和 $10 \%$ 的导电碳黑(上海杉杉科技有
限公司) 的质量百分比组成, 电解液为 $1 \mathrm{~mol} / \mathrm{L}$ $\mathrm{NaClO}_{4}-\mathrm{EC}: \mathrm{DEC}\left(\right.$ 体积比为 $1: 1$ ) 和 $1 \mathrm{~mol} / \mathrm{L} \mathrm{NaClO}_{4}$ EC:DEC:PC(体积比 $1: 1: 1)($ 安徽兆达新能源科技有限 公司). 充放电性能测试采用 2032 扣式电池, 金属钠片 作为对电极, 在新威电池检测系统(深圳新威电子仪器 公司)上完成, 充放电倍率为 $0.1 \mathrm{C}$, 截止电压为 $0 \sim 3 \mathrm{~V}$.

$\mathrm{CV}$ 和 EIS 测试采用自制三电极玻璃电解池体系, 金属钠片作为参比和辅助电极, 在电化学工作站 (CHI660C, 上海辰华仪器有限公司)上完成. 电极片大 小为 $2 \mathrm{~cm} \times 2 \mathrm{~cm}$, 电极片活性物质担载量为 $1 \mathrm{mg} / \mathrm{cm}^{2}$. $\mathrm{CV}$ 测试中扫描速度为 $1 \mathrm{mV} / \mathrm{s}$, EIS 频率扫描范围为 $10^{5} \sim 10^{-2} \mathrm{~Hz}$, 施加的交流信号振幅为 $5 \mathrm{mV}$. 电极极化 电位的改变通过电位阶跃的方法实现, 在进行阻抗测试 前, 电极在极化电位平衡 $1 \mathrm{~h}$. 实验中获得的电化学阻 抗谱用 Zview 软件进行模拟.

\section{References}

[1] Tarascon, J. M. Nat. Chem. 2010, 2, 510.

[2] Xiang, X. D.; Lu, Y. Y.; Chen, J. Acta Chim. Sinica 2017, 75, 154. (向兴德, 卢艳莹, 陈军, 化学学报, 2017, 75, 154.)

[3] Vikström, H.; Davidsson, S.; Höök, M. Appl. Energy 2013, 110, 252.

[4] Kundu, D.; Talaie, E.; Duffort, V.; Nazar, L. F. Angew. Chem., Int. Ed. 2015, 54, 3431 .

[5] Li, H.; Wang, Z.; Chen, L.; Huang, X. Adv. Mater. 2009, 21, 4593.

[6] Komaba, S.; Murata, W.; Ishikawa, T.; Yabuuchi, N.; Ozeki, T.; Nakayama, T.; Ogata, A.; Gotoh, K.; Fujiwara, K. Adv. Funct. Mater. 2011, 21, 3859 .

[7] Zhang, S. W.; Zhang, J.; Wu, S. D.; Lv, W.; Kang, F. Y.; Yang, Q. H. Acta Chim. Sinica 2017, 75, 163. (张思伟, 张俊, 吴思达, 吕伟, 康飞宇, 杨全红, 化学学报, 2017, 75, 163.)

[8] Wang, L.; Yang, G. R.; Wang, J. N.; Wang, S. L.; Peng, S. J.; Yan, W. Acta Chim. Sinica 2018, 76, 666. (王玲, 杨国锐, 王嘉楠, 王思岗, 彭生杰, 延卫, 化学学报, 2018, 76, 666.)

[9] Narayanrao, R.; Joglekar, M.; Inguva, S. J. Electrochem. Soc. 2013, 160, A125.

[10] Lin, X.; Park, J.; Liu, L.; Lee, Y.; Sastry, A.; Lu, W. J. Electrochem. Soc. 2013, 160, A1701.

[11] Pinson, M. B.; Bazant, M. Z. J. Electrochem. Soc. 2013, 160, A243.

[12] Xu, K. Chem. Rev. 2014, 114, 11503.

[13] Zhuang, Q. C.; Xu, S. D.; Qiu, X. Y.; Cui, Y. L.; Fang, L.; Sun, S. G. Prog. Chem. 2010, 22, 1044. (庄全超, 徐守冬, 邱祥云, 崔永丽, 方亮, 孙世刚, 化学进展, 2010, 22, 1044.)

[14] Qin, Y. P.; Zhuang, Q. C.; Shi, Y. L.; Jiang, L.; Sun, Z.; Sun, S. G. Prog. Chem. 2011, 23, 390. (秦银平, 庄全超, 史月丽, 江利, 孙 智, 孙世刚, 化学进展, 2011, 23, 390.)

[15] Qiu, X. Y.; Zhuang, Q. C.; Zhang, Q. Q.; Cao, R.; Ying, P. Z.; Qiang, Y. H.; Sun, S. G. Phys. Chem. Chem. Phys. 2012, 14, 2617.

[16] Zhuang, Q. C.; Wei, T.; Du, L. L.; Cui, Y. L.; Fang, L.; Sun, S. G. J. Phys. Chem. C 2010, 114, 8614.

[17] Qiu, X. Y.; Zhuang, Q. C.; Zhang, Q. Q.; Cao, R.; Qiang, Y. H.; Ying, P. Z.; Sun, S. G. J. Electroanal. Chem. 2012, 687, 35.

[18] Wei, T.; Zhuang, Q. C.; Wu, C.; Cui, Y. L.; Fang, L.; Sun, S. G. Acta Chim. Sinica 2010, 68, 1481. (魏涛, 庄全超, 吴超, 崔永丽, 方亮, 孙世刚, 化学学报, 2010, 68,1481 .)

[19] Zhuang, Q. C.; Wei, T.; Wei, G. Z.; Dong, Q. F.; Sun, S. G. Acta Chim. Sinica 2009, 67, 2184. (庄全超, 魏涛, 魏国祯, 董全峰, 孙 世刚, 化学学报, 2009, 67, 2184.)

[20] Zheng, M.; Liu, Y.; Xiao, Y.; Zhu, Y.; Guan, Q.; Yuan, D.; Zhang, J. J. Phys. Chem. C 2009, 113, 8455.

[21] Cao, Y.; Xiao, L.; Sushko, M. L.; Wang, W.; Schwenzer, B.; Xiao, J.; Nie, Z.; Saraf, L. V.; Yang, Z.; Liu, J. Nano Lett. 2012, 12, 3783.

[22] Li, Y.; Hu, Y. S.; Titirici, M. M.; Chen, L.; Huang, X. Adv. Energy Mater. 2016, 6, 1600659. 
[23] Liu, P.; Li, Y.; Hu, Y. S.; Li, H.; Chen, L.; Huang, X. J. Mater. Chem A 2016, 4, 13046.

[24] Holzapfel, M.; Martinent, A.; Alloin, F.; Le Gorrec, B.; Yazami, R.; Montella, C. J. Electroanal. Chem. 2003, 546, 41

[25] Chang, Y. C.; Sohn, H. J. J. Electrochem. Soc. 2000, 147, 50.
[26] Levi, M.; Aurbach, D. J. Phys. Chem. B 1997, 101, 4630.

[27] Xu, S. D.; Zhuang, Q. C.; Tian, L. L.; Qin, Y. P.; Fang, L.; Sun, S. G. J. Phys. Chem. C 2011, 115, 9210 .

[28] Zhuang, Q. C.; Li, J.; Tian, L. L. J. Power Sources 2013, 222, 177.

(Zhao, C.) 